\title{
La sostenibilidad en las universidades públicas valencianas: una comparativa cronológica y con otros campus españoles
}

\author{
Luisa Marti ${ }^{\mathrm{a}}$, Rosa Puertas ${ }^{\mathrm{b}}$, Consuelo Calafat ${ }^{\mathrm{c}}$ \\ ${ }^{a}$ Universitat Politècnica de València, España, email: mlmarti@esp.upv.es, ${ }^{b}$ Universitat Politècnica de \\ València, España, email: mlmarti@esp.upv.es, ' Universitat Politècnica de València, España, email: \\ mlmarti@esp.upv.es
}

\section{Resumen}

El papel que juega la universidad en la sociedad podría cuantificarse mediante los estudios de indicadores de sostenibilidad. Tanto en el ámbito nacional como internacional se están redefiniendo las estrategias de implementación de líneas de sostenibilidad a partir de programas y redes internacionales como: "Sustainable Development Solutions Network" (Naciones Unidas), The International Sustainable Campus Network, the Association for the Advancement of Sustainability in Higher Education (EEUU) o the Environmental Association for Universities and Colleges (Reino Unido). En España existe la comisión denominada "CRUESostenibilidad" donde se desarrolla la temática de la calidad ambiental y desarrollo sostenible en las universidades españolas. Dicha comisión ha creado una herramienta orientada a realizar un diagnóstico sobre la sostenibilidad ambiental en la universidad española.

Actualmente se están desarrollando, a un ritmo significativo medidas sobre sostenibilidad, haciendo necesaria su evaluación a través de herramientas concretas. El análisis que se presenta en el artículo utiliza el ranking UI GreenMetric desarrollado por la Universidad de Indonesia. En este ámbito, el objetivo del artículo es analizar los indicadores de sostenibilidad de las universidades públicas pertenecientes a la Comunidad Valenciana. Desde una perspectiva temporal, se posicionarán a los centros valencianos en el crecimiento de los indicadores en comparación con la evolución de otras universidades españolas durante el periodo 2017-2018. Además, se desarrollaran las estrategias a seguir en materia de sostenibilidad comparando el plan de actuación de unas universidades con otras. Todo ello permitirá poner en conocimiento el grado de compromiso y responsabilidad que tienen las universidades con el fin de cumplir los objetivos de desarrollo sostenible. 
La sostenibilidad en las universidades públicas valencianas: una comparativa cronológica y con otros campus españoles

Palabras clave: UI GreenMetric, Universidad publicas valencianas, sostenibilidad

\section{Introducción}

La gran actividad existente en los campus universitarios, junto con la creciente preocupación por el cambio climático ha derivado en la necesidad de analizar su impacto medioambiental, para poder mitigar los efectos adversos. En esta línea, se han desarrollado índices con la finalidad de cuantificar la contribución de estas instituciones, como Green League 2007 o Environmental and Social Responsibility Index 2009 (Grindsted, 2011). Sin embargo, estos índices no han tenido la transcendencia que se esperaba, de ahí que Univesitas Indonesia (UI) desarrolló en 2010 un ranking "green" a nivel mundial de las universidades con objeto de valorar su implicación en todos los aspectos de la sostenibilidad, denominado UI GreenMetric. Dicho ranking ha sido utilizado como instrumento de apoyo al desarrollo sostenible de los centros de educación superior, tal y como muestran los trabajos de Suwartha y Sari (2017) y Sonetti et al (2016), concretamente éste último realiza una comparativa entre una universidad italiana y otra japonesa. Más recientemente, Drahein et al (2019) utiliza el UI GreenMetric para analizar la sostenibilidad en las universidades brasileñas, mientras que Parvez y Agrawal (2019) lo hacen sobre los centros de educación superior de India.

Actualmente están aflorando una gran diversidad de instrumentos con objeto de medir la sostenibilidad, haciendo necesaria su evaluación mediante de herramientas concretas. En este ámbito, el objetivo del artículo es analizar los indicadores de sostenibilidad de las universidades públicas pertenecientes a la Comunidad Valenciana a partir del ranking UI GreenMetric. Desde una perspectiva temporal, se trata de posicionar los campus valencianos en el crecimiento de los indicadores, realizando una comparativa con la evolución de otras universidades españolas durante el periodo 2017-2018. Además, se analizarán las estrategias a seguir en materia de sostenibilidad contrastando el plan de actuación de los distintos campus. Todo ello permitirá poner en conocimiento el grado de compromiso y responsabilidad que tienen los centros de educación superior en el cumplimiento de los objetivos de desarrollo sostenible.

El resto del artículo se estructura de la siguiente forma. En la sección 2 se desarrolla la metodología aplicada por el ranking UI GreenMetric. En la sección 3 se compara la evolución temporal de las universidades españolas según los indicadores ambientales. En la sección 4 se analizan en profundidad los centros valencianos según cada uno de los componentes del UI GreenMetric. En la sección 5 se explican los planes estratégicos en 
materia ambiental de cada universidad. Por último, en la sección 6 se resumen las principales conclusiones.

\section{Metodología. Ranking UI GreenMetric}

UI GreenMetric World University Rankings fue iniciado en 2010 por Universitas Indonesia (UI) con el objetivo de poder valorar las políticas de sostenibilidad de las universidades. En 2017 se han evaluado 619 universidades repartidas por más de 76 países contemplando el marco conceptual de medio ambiente, economía y equidad, llegando a 718 en 2018 . El ranking este compuesto por distintos indicadores ponderados según relevancia:

- Infraestructuras: aporta información sobre la política de ambiental seguido por el centro. (ponderación del $15 \%$ )

- Energía y cambio climático: explora sobre aplicación de energías renovables y eficientes en los edificios universitarios, (ponderación del 21\%).

- Reciclaje: mide los programas y tratamientos de residuos implantados en los campus.

- Agua: evalúa el consumo de agua, así como los programas de conservación y protección del hábitat.

- Transporte: valora las políticas de transporte referentes a la limitación de vehículos en el campus, el fomento del servicio público y la bicicleta

- Educación e investigación: estima el papel de la universidad como centro de aprendizaje de la sociedad en temas de sostenibilidad.

Estos cuatro últimos indicadores tienen todos ellos un peso del $18 \%$ en el índice global.

\section{Evolución temporal de las universidades españolas según los indicadores ambientales}

En España, desde el 2007, un grupo de universidades trabaja en la evaluación de los campus y han establecido indicadores para determinar su aporte a la sostenibilidad. Sus resultados parciales son recogidos en el estudio de Alba et al (2012) donde concluyen que la gestión de residuos es la actuación con mayor avance entre los centros españoles. En este artículo se propone analizar el UI GreenMetric explicado en la sección 2. A continuación, 
en el Gráfico 1 se exponen los valores de la puntuación global obtenida por las 28 universidades españolas que han participado en el UI GreenMetric en 2017 y 2018.

Los resultados reflejan una creciente implicación de todas las universidades analizadas, con crecimientos comprendidos entre el $25,37 \%$ de la universidad de les Illes Balears y el 9,92\% del campus de Valladolid. Se confirma que las políticas de sostenibilidad aplicadas en España están teniendo una gran acogida en los centros de educación superior, otorgándoles una mayor competitividad tanto a nivel nacional como internacional. La Universidad de Alcalá, es la que ocupa la primera posición, cuenta con un Programa de Calidad Ambiental, un Plan integral de sostenibilidad y una Oficina de Participación, Análisis e Iniciativas Ambientales (Ecocampus); En segundo lugar, la Universidad Autónoma de Barcelona es reconocida por su innovación en la infraestructura con el uso de "piel bioclimática", que controla la temperatura interna de los edificios y reduce hasta un $62 \%$ el consumo de energía. Seguidamente, cabe destacar la tercera posición de la Universidad Autónoma de Madrid, la cual es líder por su relación de cursos y asignaturas sobre sostenibilidad, publicaciones y eventos relacionados con el medio ambiente y la cantidad de organizaciones estudiantiles que se dedican a garantizar el cuidado del entorno. En su conjunto, se puede valorar positivamente todas las actuaciones en materia de sostenibilidad que están llevando a cabo los centros de educación superior, permitiendo mejorar los valores de ranking UI GreenMetric en un $17,79 \%$ en media entre todas las universidades españolas.

Grafico 1. Resultado global del UI GreenMetric por universidades (2017-2018)

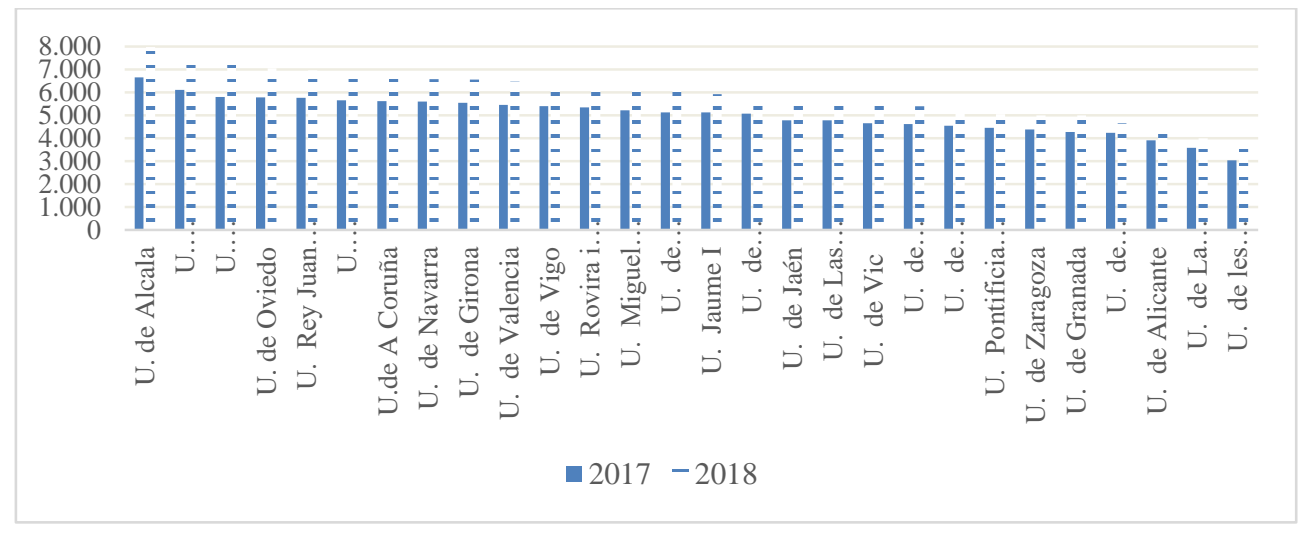

Fuente: Elaboración propia a partir de datos de UI GreenMetric 


\section{Análisis de la sostenibilidad en las universidades valencianas}

Los centros de educación superior valencianos van tomando posiciones relevantes en los temas de sostenibilidad, siendo sus prácticas fructíferas a través del reconocimiento otorgado por los rankings internaciones. Siguiendo con los objetivos de la investigación, en el Gráfico 2 se muestra el comportamiento de los 5 centros valencianos en el ranking UI GreenMetric en el periodo 2014-2018.

Gráfico 2. Evolución temporal del resultado global del UI GreenMetric en universidades públicas valencianas

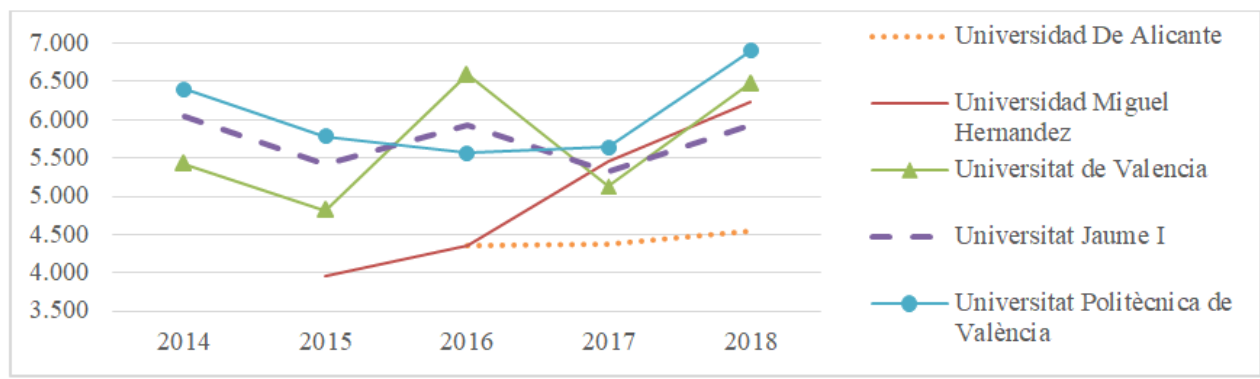

Fuente: Elaboración propia a partir de datos de UI GreenMetric

La Universitat Politècnica de València (UPV) ha liderado la primera posición del ranking en todo el periodo analizado a excepción de 2016 (Gráfico 2). Se trata de un centro donde destaca la Red de Voluntarios UPV, un Máster Universitario en Cooperación al Desarrollo de esta institución, jornadas, encuentros y congresos vinculados a la Agenda 2030, así como la apuesta de la universidad por incluir los valores de los ODS en su plan estratégico. En segundo lugar, ocupando la posición 112 del ranking, se encuentra la Universitat de València (UV). Se trata de un campus que ha decidido incluir la sostenibilidad como un eje fundamental de su estrategia, impulsando iniciativas para un desarrollo sostenible en todos los ámbitos que le corresponden. En tercer lugar, aunque algunos años ocupaba la segunda posición, la Universidad Jaume I también presenta buenos resultados en el ranking UI GreenMetric (posición 154). Este centro cuenta con una Oficina de Prevención y Gestión Medioambiental. El resto de universidades valencianas situadas fuera de la provincia de Valencia tienen unos valores de sostenibilidad menores pero con tendencia creciente en 2017 y 2018.

A continuación, en la Tabla 1 se analizan los componentes del UI GreenMetric para las cinco universidades valencianas analizadas en esta sección. El análisis por componentes indica que tanto el factor residuos como el de educación están liderados todos los años por una misma universidad, la UPV y la UV, respectivamente. En el caso de los residuos, el 
reconocimiento está avalado por las políticas de sostenibilidad y compromiso con el medio ambiente de la UPV, suponiendo un nuevo impulso para una universidad que no solo se preocupa por la búsqueda de la excelencia formativa, sino que además promueve valores y condiciones fundamentales para el presente y el futuro de la humanidad. Por otra parte, en el componente de educación ambiental la UV destaca por sus cursos y masters en materia de sostenibilidad, así como las conclusiones obtenidas en las investigaciones realizadas por el denominado "Grupo de Investigación sobre Sostenibilidad y Educación Superior”.

Tabla 1. Componentes del ranking UI GreenMetric para las universidades valencianas

\begin{tabular}{|c|c|c|c|c|c|c|}
\hline & $\begin{array}{c}\text { Situación e } \\
\text { infraestruc- } \\
\text { tura }\end{array}$ & $\begin{array}{l}\text { Energia y } \\
\text { cambio } \\
\text { climático }\end{array}$ & Residuos & Agua & Transporte & $\begin{array}{l}\text { Educación e } \\
\text { investigación }\end{array}$ \\
\hline & \multicolumn{6}{|c|}{2018} \\
\hline UPV & 700 & 1200 & 1650 & 525 & 1175 & 1650 \\
\hline UV & 825 & 975 & 1425 & 500 & 950 & 1800 \\
\hline UA & 250 & 800 & 1275 & 525 & 775 & 925 \\
\hline UJI & 1150 & 1000 & 1125 & 575 & 1125 & 950 \\
\hline \multirow[t]{2}{*}{ UMA } & 775 & 1275 & 1200 & 775 & 950 & 1250 \\
\hline & \multicolumn{6}{|c|}{2017} \\
\hline UPV & 633 & 1175 & 1701 & 383 & 913 & 835 \\
\hline UV & 640 & 895 & 1476 & 475 & 613 & 1030 \\
\hline UA & 396 & 671 & 1425 & 407 & 913 & 559 \\
\hline UJI & 834 & 958 & 1203 & 650 & 1012 & 675 \\
\hline \multirow[t]{2}{*}{ UMA } & 738 & 1224 & 1551 & 660 & 713 & 561 \\
\hline & \multicolumn{6}{|c|}{2016} \\
\hline UPV & 215 & 1093 & 1551 & 400 & 1167 & 1138 \\
\hline UV & 751 & 1031 & 1476 & 775 & 965 & 1596 \\
\hline UA & 171 & 682 & 1275 & 407 & 977 & 839 \\
\hline UJI & 919 & 870 & 1326 & 775 & 976 & 1071 \\
\hline \multirow[t]{2}{*}{ UMA } & 820 & 567 & 1224 & 10 & 913 & 827 \\
\hline & \multicolumn{6}{|c|}{2015} \\
\hline UPV & 356 & 1550 & 1725 & 700 & 762 & 689 \\
\hline UV & 532 & 1025 & 1575 & 475 & 163 & 1047 \\
\hline UA & - & - & - & - & - & - \\
\hline UJI & 420 & 875 & 1725 & 675 & 831 & 891 \\
\hline \multirow[t]{2}{*}{ UMA } & 535 & 575 & 1200 & 438 & 527 & 684 \\
\hline & \multicolumn{6}{|c|}{2014} \\
\hline UPV & 397 & 1645 & 1725 & 750 & 1350 & 529 \\
\hline UV & 565 & 1545 & 1500 & 625 & 400 & 786 \\
\hline UA & - & - & - & - & - & - \\
\hline UJI & 232 & 1385 & 1725 & 1000 & 925 & 779 \\
\hline UMA & - & - & - & - & - & - \\
\hline
\end{tabular}

Nota: UPV: Universitat Politècnica de València; UV: Universitat de València; UA: Universidad de Alicante; UJI: Universidad Jaime I, UMA: Universidad Migue Hernandez

Fuente: Elaboración propia a partir de datos de UI GreenMetric 
Los otros tres componentes no muestran la uniformidad anterior. En el factor transporte la UPV lidera 3 años y la Universidad Jaime I de Castellón el resto, en energía nuevamente la UPV obtiene mayor puntuación durante 4 años mientras que la Universidad Miguel Hernández tan sólo en 2017. La gestión del agua es el componente con un liderazgo más diversificado, se van alternando unos centros y otros siendo de nuevo la Universidad Miguel Hernández líder los dos últimos años analizados. Por último, en infraestructuras ha sido la universidad castellonense la que ha liderado dicho factor durante 3 años.

\section{Plan de actuación en materia de sostenibilidad de las universidades valencianas}

El plan de actuación constituye la mejora que deben realizar las universidades valencianas para llegar a ser más sostenibles a nivel internacional. Siguiendo con la herramienta básica del ranking UI GreenMetric se puede cuantificar el plan de actuación en materia de sostenibilidad de la siguiente forma.

Si se tiene en cuenta que el valor máximo del resultado del ranking UI GreenMetric es de 10.000 puntos, el recorrido de mejora es calculado como:

$$
\text { Recorrido de mejora }=\frac{(10000-\text { valor ranking }(i, t))}{\text { valor ranking }(i, t)} \times 100
$$

Donde "valor ranking $(i, t)$ " es la posición que ocupa la universidad " $\mathrm{i}$ " en el momento " $\mathrm{t}$ ". De esta forma el valor del recorrido de mejora está comprendido entre 0 y 100 , donde los valores menores representan universidades con un recorrido menor porque están más implicadas en todos los aspectos de la sostenibilidad. A continuación, a partir de los datos facilitados por UI GreenMetric, se han calculado los recorridos de mejora de 2017 y 2018 de las universidades valencianas (Tabla 2).

Los resultados muestran el gran esfuerzo realizado el último año por todos los campus valencianos, el recorrido de mejora de todos ellos ha disminuido significativamente. En primera posición se encuentra la UPV con un recorrido de $44,9 \%$, lo que implica necesita mejorar un $45 \%$ para ocupar los primeros del ranking UI GreenMetric. En el extremo se encuentra la UA, que a pesar de los esfuerzos realizados el último año, debe más que duplicar sus esfuerzos para llegar a dicho objetivo. En definitiva, se puede afirmar que los centros valencianos están cada vez más implicados en todos los aspectos de la 
La sostenibilidad en las universidades públicas valencianas: una comparativa cronológica y con otros campus españoles

sostenibilidad, siendo latente su empeño en mejorar e ir escalando posiciones en los rankings internacionales.

Tabla 2. Evolución del recorrido de mejora de las universidades valencianas

\begin{tabular}{|l|c|c|}
\hline & Recorrido mejora 2017 & Recorrido mejora 2018 \\
\hline UPV & $77,3 \%$ & $44,9 \%$ \\
\hline UV & $83,6 \%$ & $54,4 \%$ \\
\hline UMH & $92,0 \%$ & $60,6 \%$ \\
\hline UJI & $95,3 \%$ & $68,8 \%$ \\
\hline UA & $155,8 \%$ & $119,8 \%$ \\
\hline
\end{tabular}

Fuente: Elaboración propia

\section{Conclusiones}

Los centros de educación superior están aportando su granito de arena para crear un mundo más respetuoso con el medio ambiente y reducir la huella ecológica de los consumidores, destacando algunos de ellos por sus iniciativas y logros. En este artículo se ha tratado de realizar un análisis comparativo entre las universidades españoles, haciendo especial referencia a los ubicados en la Comunidad Valenciana. El ranking UI GreenMetric publicado por la Universidad de Indonesia ha sido la herramienta base en la realización de la investigación. Según el Grupo de trabajo sobre Evaluación de la Sostenibilidad Universitaria perteneciente a la CRUE (2018), todos los campus tienen responsables políticos en materia de medio ambiente y se ha alcanzado un control de los aspectos ambientales, agua, energía y residuos, existiendo una falta sistemática de la implementación de acciones de mejora para los mismos. En este artículo también se ha constatado que las universidades valencianas han sabido liderar su gestión de energía, agua, residuos, educación e infraestructura en algún momento del tiempo.

Sin embargo, el plan de actuación de los centros valencianos debe estar enfocado a mejorar todos los aspectos de la sostenibilidad, siendo la UA la que mayores esfuerzos debe hacer. En el otro extremo se encuentra la UPV, se trata de la universidad valenciana mejor valorada por el UI GreenMetric 2018, teniendo menor recorrido de mejora que el resto de campus valencianos para ocupar puestos relevantes a nivel internacional. 


\section{Referencias}

Alba, D., Barbeitos R., Barral, M.T., Benayas, J., Blanco, D., Domènech, X., Fernández, I., Florensa, A., García, F., López, N., Ysern, P. (2012). Estrategias de sostenibilidad y responsabilidad social en las universidades españolas: una herramienta para su evaluación. Profesorado. Revista de curriculum y formación del profesorado, 16(2), 59- 70.

CRUE (2018). Diagnostico de la sostenibilidad ambiental en las universidades españolas" Grupo de trabajo sobre evaluación de la sostenibilidad universitaria. Informe CRUE.

Drahein, AD., De Lima, EP., Da Costa, SE. (2019). Sustainability assessment of the service operations at seven higher education in Brazil. Journal of Cleaner Production, 212, 527-536.

Grindsted, T.S. (2011). Sustainable universities -from declarations on sustainability in higher education to national law. Journal of Environmental Economics, 2 (2), 29-36.

Parvez, N., Agrawal, A. (2019). Assessment of sustainable development in technical higher education institutes of India. Journal of Cleaner Production, 214, 975-994.

Sonetti, G., Lombardi, P., Chelleri, L. (2016). True Green and Sustainable University Campuses? Toward a Clusters Approach. Sustainability, 8(83), 1-23.

Suwartha, N., Sari, RF. (2017). Evaluating UI GreenMetric as a tool to support green universities development: assessment of the year 2011 ranking. Journal of Cleaner Production, 61, 46-53.

UI GreenMetric (2018). Guideline of UI GreenMetric World University Ranking, 2018. Universitas Indonesia. 